\title{
FAST AND ROBUST SEGMENTATION AND CLASSIFICATION FOR CHANGE DETECTION IN URBAN POINT CLOUDS
}

\author{
X. Roynard ${ }^{\mathrm{a}, *}$ J-E. Deschaud ${ }^{\mathrm{a}}$, F. Goulette ${ }^{\mathrm{a}}$ \\ ${ }^{a}$ MINES ParisTech, PSL Research University, Centre for robotics, 60 Bd St Michel 75006 Paris, France \\ (xavier.roynard, jean-emmanuel.deschaud, francois.goulette)@ mines-paristech.fr
}

Commission III, WG III/4

KEY WORDS: Urban Point Cloud, Mobile Laser Scanning, Segmentation, Classification, 3D-Features

\begin{abstract}
:
Change detection is an important issue in city monitoring to analyse street furniture, road works, car parking, etc. For example, parking surveys are needed but are currently a laborious task involving sending operators in the streets to identify the changes in car locations. In this paper, we propose a method that performs a fast and robust segmentation and classification of urban point clouds, that can be used for change detection. We apply this method to detect the cars, as a particular object class, in order to perform parking surveys automatically. A recently proposed method already addresses the need for fast segmentation and classification of urban point clouds, using elevation images. The interest to work on images is that processing is much faster, proven and robust. However there may be a loss of information in complex 3D cases: for example when objects are one above the other, typically a car under a tree or a pedestrian under a balcony. In this paper we propose a method that retain the three-dimensional information while preserving fast computation times and improving segmentation and classification accuracy. It is based on fast region-growing using an octree, for the segmentation, and specific descriptors with Random-Forest for the classification. Experiments have been performed on large urban point clouds acquired by Mobile Laser Scanning. They show that the method is as fast as the state of the art, and that it gives more robust results in the complex 3D cases.
\end{abstract}

\section{INTRODUCTION}

With the recent availability of massive urban point clouds, we see an interest in automatic segmentation and classification of this data for numerous tasks dealing with city analysis and surveying. Such as emergency simulation, accessibility analysis, city modelling, itinerary planning, maintenance, all kind of network management (rails, road ,electricity, telecommunication, water, gas, etc).

Then we need to extract semantic data of the point clouds, what can be summarized in giving a segmentation and a classification of the point clouds in relevant objects. And for the application of change detection, being able to compare different segmented clouds. In the case of parking surveys, we must detect and segment sub-clouds that represent parked cars, then compare them between different passages.

There are already methods (Serna and Marcotegui, 2014) that automatically segment and classify urban point clouds in reasonable time. But this methods project the point clouds in 2D and lose a lot of information, as cars under trees or pedestrians under balcony.

We propose an automatic algorithm to segment and classify urban point clouds in similar processing time, but keeping 3D information and then with more robustness.

The point cloud is projected on a 2D-grid for ground extraction by region-growing. Then we project the cloud on a 3D-grid for object segmentation. We calculate 3D descriptors on each segmented object. Finally using a Random-Forest model to perform classification.

\footnotetext{
${ }^{*}$ Corresponding author
}

Our method has enabled us to create a sufficiently large dataset to test the classification algorithm. We test the classification performance and the importance of each descriptor used. In addition we measure the computation time for each step of the method to identify bottlenecks.

This paper is organized as follows. Section 2. reviews related work in the state of the art. Section 3. explains our methodology. Section 4. describes the experiments we used to give the results in section 5., and conclude in section 6 .

\section{RELATED WORK}

There are multiple methods of segmentation and classification of urban point clouds, but we can at first distinguish between methods using a 2D information (either by projecting the cloud of an elevation image, or directly using range images) and methods working directly on the $3 \mathrm{D}$ information. Also the way the two steps segmentation and classification can combine may differ greatly from one method to another. Indeed, one can start by segmenting objects and classify each object, but one can also classify each point and use this classification to group points into "real" objects.

Elevation images: The use of images reduces the complexity and therefore the computation time, and use proven and robust methods of image processing (Hoover et al., 1996). It can go from the simplest with the research plans in range images(Gorte, 2007), to semantic segmantation with graphs and CART decision trees on range images (Zhu et al., 2010), or mathematical morphology for segmentation and SVM for clssification (Serna and Marcotegui, 2014). Elevation images are also used for guiding 
autonomous vehicles in real-time, indeed they allow for fast registration on pre-processed 2D maps (Kammel et al., 2008, Ferguson et al., 2008). Markov Random Fields can be used for contextual classification (Munoz et al., 2009), or for learning segmentation (Anguelov et al., 2005).

Direct point classification: Another approach is to first classify each point by studying its neighbourhood, then group them into relevant objects. This raises the problem of choosing the size of the neighbourhood. This problem is addressed in (Demantké et al., 2011) by choosing the size that minimizes an entropy computed on features extracted from covariance matrix of the neighbourhood called dimensionality features. This method proposes to classify each point in a geometric class: line, surface, volume. This dimensionality attributes are used in several methods (Douillard et al., 2011). There are also many local 3D descriptors as VFH estimating normal distributions (Rusu et al., 2010) or RSD using the curvature radius(Marton et al., 2010a) that can be used to build global descriptors of objects CVFH (Aldoma et al., 2011) and GRSD (Marton et al., 2010b).

Detection, Segmentation, Classification: The pipeline which seems the most successful is the following (Golovinskiy et al., 2009): a) ground extraction, b) object detection, c) object segmentation, d) supervised learning classification. It was improved by (Velizhev et al., 2012) with object centres estimation and Implicit Shape Model for classification instead of supervised learning.

\section{PROPOSED METHODOLOGY}

In a framework inspired by (Golovinskiy et al., 2009), we propose the following workflow:

- extract the ground,

- segment the remaining 3D point cloud in connected components (no detection step, that appears to be unnecessary in three dimensions),

- compute a feature vector on each segmented object with 3D descriptors,

. classify each object.

\subsection{Ground Extraction}

After testing several methods including RANSAC and RegionGrowing on normal distribution, we chose the following method. We project the point cloud on an horizontal grid discretized with a step $\delta_{x y}$, keeping in each pixel $\mathcal{P}$ only the minimal applicate value: $z_{\min }(\mathcal{P})=\min _{p \in \mathcal{P}} p_{z}$.

First, we find a pixel which has supposedly a lot of his points on the ground. For this purpose, we compute an histogram in $z$ of the whole point cloud and we find the biggest bin, then we find the pixel of the 2D-grid which has the most points in this bin in proportion to all its points.

Then we grow a region $\mathcal{R}$ from this seed, adding a neighbour $\mathcal{Q}$ of $\mathcal{P} \in \mathcal{R}$ to the region only if $\left|z_{\text {min }}(\mathcal{P})-z_{\text {min }}(\mathcal{Q})\right|<\delta_{z}$, where $\delta_{z}$ is a parameter to be chosen big enough to allow a certain slope of the ground, but small enough to avoid taking cars and buildings.

The last step is to filter the points in the region $\mathcal{R}$, indeed, in chosen pixels there might be remaining points not belonging to the ground. That's why we keep only points $p \in \mathcal{P}$ which verify $p_{z}<z_{\min }(\mathcal{P})+\Delta$ in each pixel. Where $\Delta$ is a threshold which must be bigger than twice the noise of the data around plane.

\subsection{Object Segmentation}

To segment the objects, we extract the connected components of the remaining cloud. As we seek to maintain reasonable computation times, we avoid to achieve a Region Growing directly on the point cloud, what is very time-consuming because of the nearest-neighbour search. Instead, we project the point cloud on a 3D-occupancy-grid or an Octree of resolution $\delta$ to be chosen large enough to avoid over-segmentation, and small enough to avoid several objects to be segmented together (for example cars parked too close to each other). Finally we realize a RegionGrowing on the occupancy-grid, it is much faster as the neighbourhoods are known in this structure.

In our experiments, it seems that optimal choice of $\delta$ is always between $10 \mathrm{~cm}$ and $15 \mathrm{~cm}$.

\subsection{Object Classification}

3.3.1 Features: For each segmented object we compute a vector of features composed of Geometric features mainly inspired by (Serna and Marcotegui, 2014) and of 3 histogram-based descriptors from the literature, namely CVFH (Aldoma et al., 2011), GRSD (Marton et al., 2010b) and ESF (Wohlkinger and Vincze, 2011). In summary we obtain a feature vector with 991 variables: 22 for geometric features, 308 for CVFH, 21 for GRSD and 640 for ESF.

Geometric features: This features are mainly inspired by (Serna and Marcotegui, 2014), they give more robustness to histogrambased descriptor which describe only the local geometry of objects.

First of all, we project horizontally the point cloud on its $(x, y)$ variables to compute the convex hull in $2 \mathrm{D}$ and covariance matrix of the point cloud in variables $(x, y)$. Then we compute the following geometric features:

. the number of points,

- $z_{\max }-z_{\min }$

- $\sigma_{z}$ the standard deviation in $z$

- $x_{\max }-x_{\min }$, in the PCA base,

- $y_{\max }-y_{\min }$, in the PCA base,

- the smallest eigenvalue $\lambda_{0}$ of covariance matrix in $(x, y)$,

- the biggest eigenvalue $\lambda_{1}$ of covariance matrix in $(x, y)$,

- $\frac{\lambda_{1}}{\lambda_{0}}$

- the area of the 2D convex hull in $(x, y)$,

- the area of the 3D convex hull,

. the volume of the 3D convex hull,

- a 10-bins histogram in $z$.

The 2 following descriptors are histogram-based originally used to recognize objects and their 6DOF-pose of known models, but we use it only for the first task. They both need normal computation, what can be very time-consuming.

CVFH (Clustered Viewpoint Feature Histogram): VFH descriptor (Rusu et al., 2010) is a concatenation of 4 histograms representing normal angles distribution on the surface of the object. As VFH is sensitive to noise, CVFH is build by adding a Shape Distribution histogram that can differentiate the clouds that have similar normal distributions, but the points are distributed differently.

GRSD (Global Radius-based Shape Descriptor): RSD (Marton et al., 2010a) descriptor is based on the observation that the radius $r$ of a sphere approximating the surface through two points at distance $d$ can be estimated using the formula $d=\sqrt{2} r \sqrt{1-\cos (\alpha)}$, 
where $\alpha$ is the angle between the normals of the points. This feature seems enough discriminative to distinguish plane, sphere, corner, edge and cylinders. But instead of using the radius $r$, RSD is the histogram in $d$ and $\alpha$ that can be used to classify each point of the object.

To build a feature vector globally on the object, the point cloud is projected on an octree, and each voxel is classified using RSD. GRSD is build as GFPFH (Rusu et al., 2009) by observing relationships between this locally labelled voxels.

ESF (Ensemble of Shape Functions): ESF descriptor is based on the idea that the distribution of distances between pairs of points randomly chosen in the object appears to be relevant to classify an object (Osada et al., 2001). Other distributions are important as angles, surfaces and volumes. To further improve performance, each pair of points is classified in "in"', "out"” or "mixed"" depending on whether the segment between these points is inside the object, outside or both. A great benefit of this descriptor is that it does not require the computation of normals, and is therefore much faster than the previous two descriptors.

3.3.2 Classifier: In our work, Random-Forest (Breiman, 2001) are chosen because they are one of the most accurate general purpose classifier and have multiple advantages compared to SVM for example, with similar results (Mountrakis et al., 2011) or Gaussian Mixture Model (Lalonde et al., 2005), Markov Random Fields (Munoz et al., 2009) and Adaboost (Lodha et al., 2007).

Random-Forest are part of variance reduction methods by bagging (bootstrap aggregating). The principle is to train independently a big number of decision tree, each one on a random subset of the training set (typically two third), and using each time only a random subset of features. Then, in testing time to obtain a prediction on the forest, simply test on each tree and count the number of votes for classification (or average for regression).

The greatest advantages of Random-Forest are:

- they give an estimation of error of generalization (out-ofbag: OOB) after training, as each tree is trained only on two third of the dataset, the model can be tested on the last third,

- for each feature vector they give the probability to be in each class,

- they give importance of each feature, measuring the number of times they are used for training, this allows us to achieve feature selection and potentially reduce computation time,

- they are poorly sensitive to parameter changes, what makes them very easy to tune.

\section{EXPERIMENTS}

\subsection{Implementation}

All algorithms are implemented in C++ with an intensive use of PCL (Rusu and Cousins, 2011) to read and write PLY files, to compute normals and to compute descriptors such as CVFH, GRSD and ESF. The framework run on one thread except the computation of normals which is parallelised with OpenMP.

For classification, computations are less intensive we use the python library scikit-learn (Pedregosa et al., 2011), which provides all features of Random-Forest as OOB score and features importance.
The experiments are run on a desktop Intel Core i7 4790K with 4 cores clocked at $4 \mathrm{GHz}$.Moreover the computer has $32 \mathrm{~GB}$ of RAM, what allows us to run our algorithms on massive point clouds of more than 100 million points.

\subsection{Dataset}

All point clouds used for our experiments are acquired with the MLS prototype of the center for robotics of Mines Paritech: L3D2 (Goulette et al., 2006) (as seen in Figure 1). It is a Citroën Jumper equipped with a GPS (Novatel FlexPak 6), an IMU (Ixsea PHINS in LANDINS mode) and a Velodyne-HDL32 LiDAR mounted in almost horizontal position.

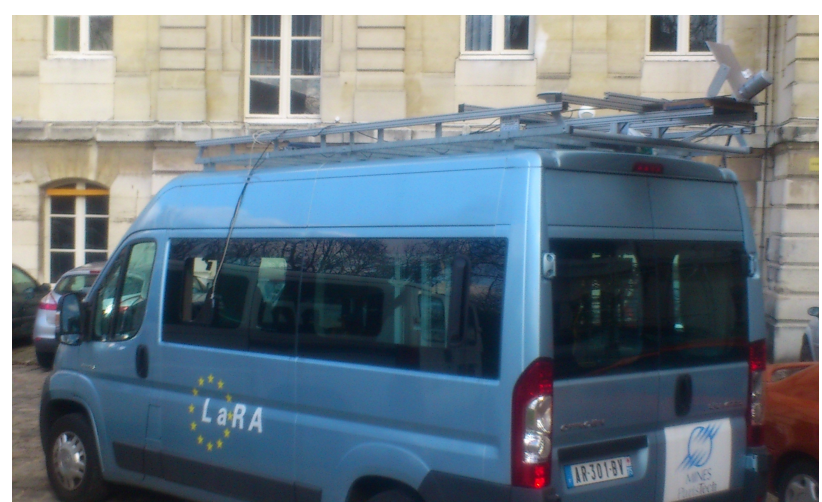

Figure 1: MLS prototype: L3D2

The Dataset is composed of seven pass of $650 \mathrm{~m}$ in Paris ${ }^{1}$ (rue Madame and rue Cassette, 75006 Paris) and seven pass of $1.6 \mathrm{~km}$ in Lille ${ }^{2}$ (rue du Professeur Perrin and rue Jean Jaurès, 59170 Croix) at about two hours apart. The clouds have high density with between 1000 and 2000 points per square meters on the ground, but there are some anisotropic patterns due to the multibeam LiDAR sensor as seen in Figure 2.

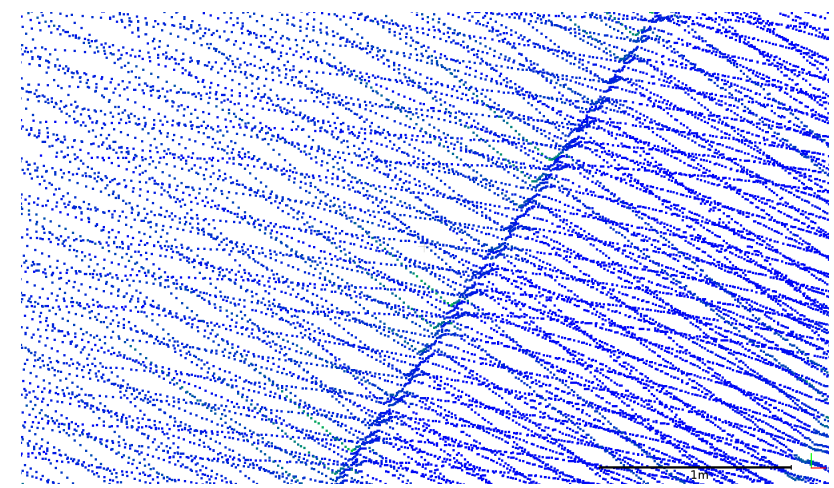

Figure 2: Anisotropic pattern on the ground (color of points is the reflectance)

${ }^{1}$ https://www.google.fr/maps/dir/48.8512675, 2 . 3320095/48.8509089, 2.3307348/@48.8496176, 2.3311634, $466 \mathrm{~m} /$ data $=$ ! 3m 1 ! $1 \mathrm{e} 3$ ! 4m9 ! 4m8! 1m5 ! 3m4! 1m2 ! 1d2.3313488! 2d48.8483308! 3s0x47e671d0662c1f71: 0x3468592da4fea104! $1 \mathrm{~m} 0 ! 3 \mathrm{e} 0 ? \mathrm{hl}=\mathrm{en}$

2 https://www .google.fr/maps/dir/50.6797937, 3 . $1565797 / 50.6719983,3.1448871 / 50.6749018,3.1456907 /$ $50.6719983,3.1448871 / @ 50.6724002,3.1451847,18.75 \mathrm{z} /$ data $=! 4 \mathrm{~m} 2 ! 4 \mathrm{~m} 1$ ! $3 \mathrm{e} 0$ 


\section{RESULTS}

\subsection{Evaluation: Segmentation}

Ground Extraction: The ground extraction step works well on all the dataset, in the sense that the road and the sidewalk is completely extracted. But an inherent consequence of our method is that the lowest points of each object are classified as belonging to the ground (as seen in figure 3(d)).

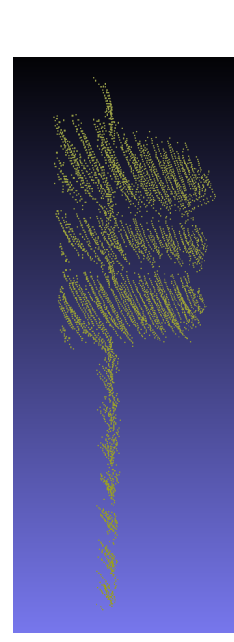

(a) raod sign

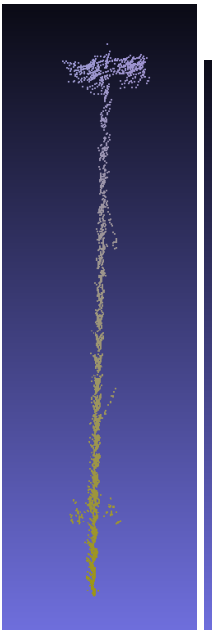

(b) lamppost

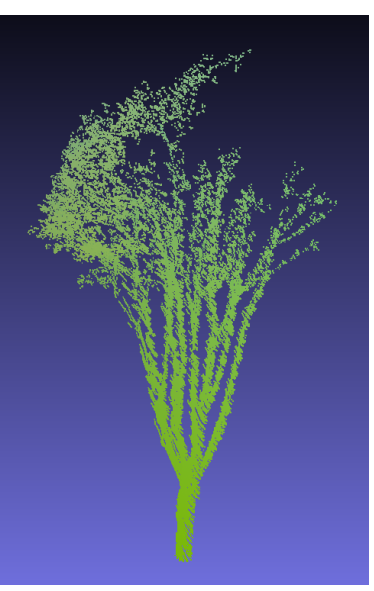

(c) tree

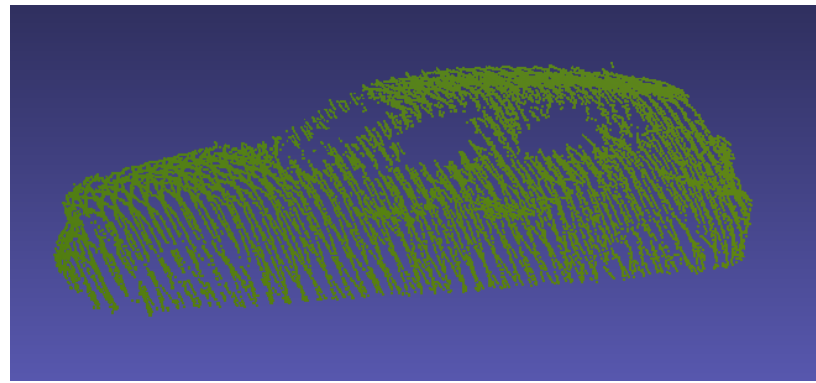

(d) car

Figure 3: Exemple of sub-clouds well segmented after ground extraction

Object Segmentation: There are a lot of artefacts: small subclouds that doesn't represent objects, for example in tree canopy or behind the buildings (as seen in figure 4). We won't use this artefacts in the Dataset for classification results, but in view of giving a class to each point of the point cloud, we should add a class "artefacts".

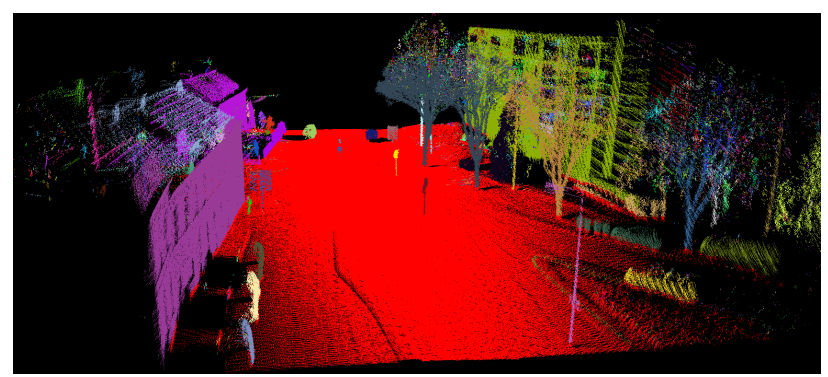

Figure 4: Exemple of ground extraction and object segmentation on a truncated point cloud of Lille (ground in red, and every other object has a different color)

We segment the whole dataset and obtain 20121 objects, most of them being artefacts. Then we classify by hand a few objects that seem well segmented, to train a first time on classifier which will give us class of other samples when it is confident enough. This allows us to create a dataset quickly. We finally have 3906 samples in 9 classes, but only 7 of them have enough samples to evaluate classification as seen in Table 1 .

\begin{tabular}{cccc}
\hline \multirow{2}{*}{ Class } & \multicolumn{3}{c}{ Number of samples } \\
\cline { 2 - 4 } & Paris & Lille & Total \\
\hline trees & 64 & 373 & 437 \\
\hline cycles & 142 & 1 & 143 \\
\hline buildings & 111 & 404 & 515 \\
\hline lampposts & 16 & 357 & 373 \\
\hline pedestrians & 10 & 1 & 11 \\
\hline poles & 508 & 65 & 573 \\
\hline trash cans & 8 & 1 & 9 \\
\hline road signs & 114 & 456 & 570 \\
\hline cars & 719 & 556 & 1275 \\
\hline Total & 1692 & 2214 & 3906 \\
\hline
\end{tabular}

Table 1: Number of samples for each class

\subsection{Evaluation: Classification}

In Random-Forest the only parameter that can influence classification is the number of trees. Figure 5 shows that OOB error converges when number of trees increases. The choice of 256 trees in the Random-Forest is made because more trees would increase computation time without real improvement in accuracy, and less trees would decrease accuracy too much.

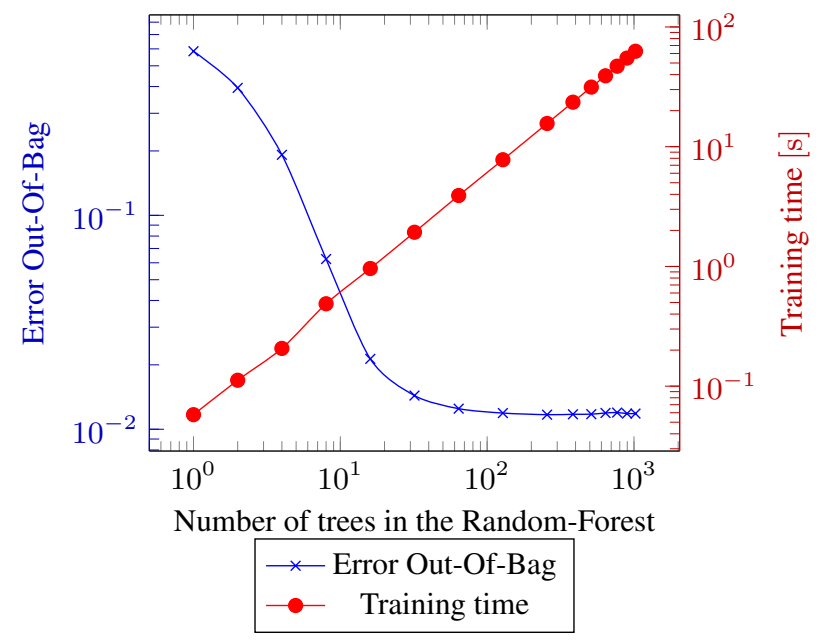

Figure 5: Importance of number of trees in the forest

To evaluate our choices in term of classification we compute 4 indicators that are the precision $P$, the recall $R$, the F1 score and the Matthews Correlation Coefficient $M C C$ as computed in equations 1 .

$$
\begin{aligned}
P & =\frac{T P}{T P+F P} \\
R & =\frac{T P}{T P+F N} \\
F 1 & =\frac{2 T P}{2 T P+F P+F N} \\
M C C & =\frac{T P \cdot T N-F P \cdot F N}{\sqrt{(T P+F P)(T P+F N)(T N+F P)(T N+F N)}}
\end{aligned}
$$


where $T P, T N, F P$ and $F N$ are respectively the number of true-positives, true-negatives, false positives and false negatives.

We train the Random Forest Model with 256 trees on the well segmented object in 7 classes that have a sufficient number of samples: trees, cycles, buildings, lampposts, poles, road signs and cars. For training we use $90 \%$ of the dataset in each class, then we compute precision, recall, $\mathrm{F} 1$ and $\mathrm{MCC}$ on the remaining $10 \%$, results are shown in table 2 .

\begin{tabular}{ccccc}
\hline Class & Precision & Recall & F1 & MCC \\
\hline trees & $93.90 \%$ & $95.84 \%$ & $94.83 \%$ & $94.19 \%$ \\
\hline cycles & $79.10 \%$ & $96.00 \%$ & $86.45 \%$ & $86.44 \%$ \\
\hline buildings & $93.00 \%$ & $98.69 \%$ & $95.73 \%$ & $95.13 \%$ \\
\hline lampposts & $90.09 \%$ & $99.00 \%$ & $94.28 \%$ & $93.79 \%$ \\
\hline poles & $92.98 \%$ & $99.69 \%$ & $96.19 \%$ & $95.60 \%$ \\
\hline road signs & $93.09 \%$ & $99.40 \%$ & $96.12 \%$ & $95.52 \%$ \\
\hline cars & $96.97 \%$ & $99.53 \%$ & $98.23 \%$ & $97.37 \%$ \\
\hline
\end{tabular}

Table 2: Mean Precision, Recall, F1 and MCC scores for each class

\subsection{Feature importances}

Random-Forest provide an importance for each feature that represent how much it is used to build the model. The importance is shown in figure 6 and table 3 for each descriptor. First of all, it seems that CVFH is almost useless both in cumulative and proportionally to number of variables. Although Geometric Features and GRSD have less variables than other descriptors they seem very important. Moreover the descriptor which has the most importance cumulatively is ESF, but importance is spread over all its 640 variables.

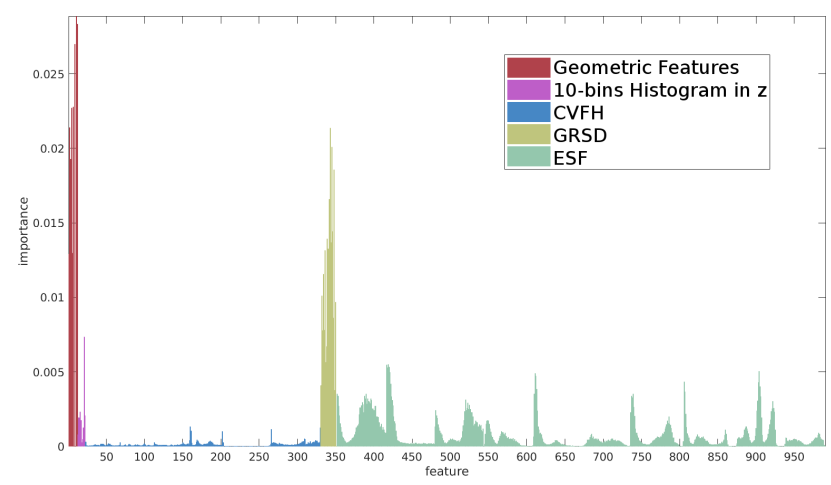

Figure 6: Features Importance

\begin{tabular}{ccc}
\hline Descriptor & $\begin{array}{c}\text { Cumulated } \\
\text { importance }\end{array}$ & $\begin{array}{c}\text { Mean importance } \\
\text { per variable }\end{array}$ \\
\hline $\begin{array}{c}\text { Geometric } \\
\text { features }\end{array}$ & $21.45 \%$ & $1.79 \%$ \\
\hline $\begin{array}{c}\text { 10-bins } \\
\text { histogram in } z\end{array}$ & $2.01 \%$ & $0.20 \%$ \\
\hline CVFH & $3.93 \%$ & $0.01 \%$ \\
\hline GRSD & $23.09 \%$ & $1.10 \%$ \\
\hline ESF & $49.52 \%$ & $0.08 \%$ \\
\hline Total & $100 \%$ & $0.10 \%$ \\
\hline
\end{tabular}

Table 3: Cumulated importance of each descriptor and Mean importance per variable (given by Randon-Forest).
To further investigate the importance of each descriptor we test every possible combination, and draw performance of classification for class "car" in table 4.

We can conclude that if we want to keep only descriptor which don't require normal computation: Geometric Features and ESF, we loose only $0.21 \%$ of OOB score. This is a compromise to be done as the normals are the most time consuming part of the computation of descriptors (as seen in 5.4).

\subsection{Computation Time}

5.4.1 Segmentation: This corresponds to the time of projection of the point cloud on a 2D-grid and a region growing on this grid for Ground Extraction, then the same thing on a 3D-grid for Object Segmentation. On our dataset, we measure similar times for Ground Extraction and Object Segmentation around 25s for sections of 10 million points. However, the 3D-grid structure has not it been optimized (e.g. using a Octree), we can think that the results could be better.

5.4.2 Descriptors: The time to compute a feature vector highly depends on the number of points of the object, that's why we give the mean computational time for each descriptor on the $20121 \mathrm{ob}-$ jects segmented in Paris and Lille Dataset.

Two descriptors require the computation of normals on the object, and the operation can be very-time consuming (as shown in table 5) especially for large clouds.

5.4.3 Classification: It seems that the time to predict the class of a feature vector is weakly dependent on the number of variables. Once the Random Forest is trained with 256 trees, prediction time per sample is about $1.2 \mathrm{~ms}$. Thus assuming that feature vectors are computed fast enough, we could predict the class of 833 objects each second. Hence the classification is clearly not the bottleneck in our algorithm.

\subsection{Comparison with state-of-the-art}

For segmentation, keeping the 3D structure of the point clouds allows to segment vehicles under trees as shown in Figure 7. What was not possible with elevation images.

Purely on the classification of cars, we compared with (Serna and Marcotegui, 2014). We obtain a better Recall, but Precision slightly below as seen in Table 6 .

\section{CONCLUSION}

In this work, we proposed an automatic and robust approach to segment and classify massive urban point clouds in reasonable time without using elevation images.

First, the ground is extracted using a Region Growing on $z_{\min }$ kept in an horizontal 2D-grid. Next, the remaining point cloud is segmented in connected components of a 3D-occupancy-grid. Finally, each object is classified using Random-Forest and a feature vector composed of geometric features and histogram-based features of the literature.

The method is tested on a Dataset acquired by a MLS prototype in Lille and Paris. Ground extraction and object segmentation is evaluated qualitatively and performs well. The classification is tested only on well-segmented objects and performs as well as the 


\begin{tabular}{|c|c|c|c|c|c|c|c|}
\hline \multirow{2}{*}{ Descriptors } & \multirow{2}{*}{$\begin{array}{c}\text { Number } \\
\text { of variables }\end{array}$} & \multirow{2}{*}{$\begin{array}{l}\text { Training } \\
\text { time (s) }\end{array}$} & \multirow{2}{*}{ OOB } & \multicolumn{4}{|c|}{ Performances for class "cars" } \\
\hline & & & & Precision & Recall & F1 & MCC \\
\hline Geom & 22 & 2.03 & $98.30 \%$ & $96.19 \%$ & $99.02 \%$ & $97.58 \%$ & $96.40 \%$ \\
\hline CVFH & 308 & 6.98 & $93.71 \%$ & $86.06 \%$ & $97.54 \%$ & $91.42 \%$ & $87.28 \%$ \\
\hline GRSD & 21 & 1.74 & $94.38 \%$ & $86.59 \%$ & $97.83 \%$ & $91.84 \%$ & $87.91 \%$ \\
\hline ESF & 640 & 13.13 & $94.29 \%$ & $87.90 \%$ & $96.02 \%$ & $91.76 \%$ & $87.71 \%$ \\
\hline Geom+CVFH & 330 & 6.34 & $97.81 \%$ & $94.32 \%$ & $99.03 \%$ & $96.60 \%$ & $94.97 \%$ \\
\hline Geom+GRSD & 43 & 2.58 & $98.39 \%$ & $96.15 \%$ & $99.44 \%$ & $97.76 \%$ & $96.68 \%$ \\
\hline Geom+ESF & 662 & 11.60 & $98.59 \%$ & $96.33 \%$ & $99.38 \%$ & $97.82 \%$ & $96.77 \%$ \\
\hline $\mathrm{CVFH}+\mathrm{GRSD}$ & 329 & 6.63 & $97.58 \%$ & $93.38 \%$ & $99.40 \%$ & $96.28 \%$ & $94.51 \%$ \\
\hline $\mathrm{CVFH}+\mathrm{ESF}$ & 948 & 15.16 & $96.04 \%$ & $91.91 \%$ & $97.57 \%$ & $94.64 \%$ & $92.03 \%$ \\
\hline GRSD+ESF & 661 & 11.83 & $98.49 \%$ & $96.17 \%$ & $99.60 \%$ & $97.85 \%$ & $96.82 \%$ \\
\hline $\mathrm{CVFH}+\mathrm{GRSD}+\mathrm{ESF}$ & 969 & 14.18 & $98.60 \%$ & $96.28 \%$ & $\mathbf{9 9 . 6 6} \%$ & $97.93 \%$ & $96.94 \%$ \\
\hline Geom+GRSD+ESF & 683 & 11.74 & $98.73 \%$ & $96.98 \%$ & $99.41 \%$ & $98.18 \%$ & $97.30 \%$ \\
\hline Geom+CVFH+ESF & 970 & 13.89 & $98.67 \%$ & $96.57 \%$ & $99.48 \%$ & $98.00 \%$ & $97.03 \%$ \\
\hline Geom+CVFH+GRSD & 351 & 6.32 & $98.18 \%$ & $95.59 \%$ & $99.23 \%$ & $97.36 \%$ & $96.09 \%$ \\
\hline Geom+CVFH+GRSD+ESF & 991 & 13.61 & $\mathbf{9 8 . 8 0} \%$ & $96.94 \%$ & $99.55 \%$ & $98.23 \%$ & $\mathbf{9 7 . 3 7 \%}$ \\
\hline
\end{tabular}

Table 4: Performances for each combination of descriptor possible (Geom is for Geometric features, for each column the best result is in bold)

\begin{tabular}{cccc}
\hline Descriptors & Computation Time (s) & Proportion & Mean Time per object (ms) \\
\hline Geom & 17.2 & $3.22 \%$ & 0.9 \\
\hline CVFH & 240.3 & $44.92 \%$ & 11.9 \\
\hline GRSD & 64.4 & $12.04 \%$ & 3.2 \\
\hline ESF & 213.0 & $39.82 \%$ & 10.6 \\
\hline Total & 534.9 & $100 \%$ & 26.6 \\
\hline \hline Normales & 964.7 & & 47.9 \\
\hline
\end{tabular}

Table 5: Mean Computational Time of descriptors on 20121 segmented objects of 7 passes in Lille and Paris

\begin{tabular}{cccc}
\hline Method & Precision & Recall & F1 \\
\hline $\begin{array}{c}\text { (Serna and } \\
\text { Marcotegui, 2014) }\end{array}$ & $\mathbf{1 0 0 . 0} \%$ & $94.6 \%$ & $97.2 \%$ \\
\hline ours & $96.97 \%$ & $\mathbf{9 9 . 5 3} \%$ & $\mathbf{9 8 . 2 3} \%$ \\
\hline
\end{tabular}

Table 6: Classification results for class "car" (in bold the best results)

state of the art. Moreover, we conduct a study of the importance of each descriptor, in order to reduce computation time.

With such classification results, it is possible to achieve change detection on particular classes such as cars. Then between two passages in the same street, we can detect cars that have changed by comparing the corresponding sub-clouds and achieve parking survey. On an other scale of time between acquisitions we can automatically detect the bent poles or road signs.

In our future works, we believe we can improve the accuracy by adding contextual descriptors (such as the elevation of objects above the ground or the number of neighbouring objects) that would avoid classifying as car objects that are in height and remove segmentation artefacts behind the buildings facades.

\section{REFERENCES}

Aldoma, A., Vincze, M., Blodow, N., Gossow, D., Gedikli, S., Rusu, R. B. and Bradski, G., 2011. Cad-model recognition and 6dof pose estimation using 3d cues. In: Computer Vision Workshops (ICCV Workshops), 2011 IEEE International Conference on, IEEE, pp. 585-592.

Anguelov, D., Taskarf, B., Chatalbashev, V., Koller, D., Gupta, D., Heitz, G. and Ng, A., 2005. Discriminative learning of markov random fields for segmentation of $3 \mathrm{~d}$ scan data. In: Computer Vision and Pattern Recognition, 2005. CVPR 2005. IEEE Computer Society Conference on, Vol. 2, IEEE, pp. 169-176.

Breiman, L., 2001. Random forests. Machine learning 45(1), pp. 5-32.

Demantké, J., Mallet, C., David, N. and Vallet, B., 2011. Dimensionality based scale selection in $3 \mathrm{~d}$ lidar point clouds. The International Archives of the Photogrammetry, Remote Sensing and Spatial Information Sciences 38(Part 5), pp. W12.

Douillard, B., Underwood, J., Kuntz, N., Vlaskine, V., Quadros, A., Morton, P. and Frenkel, A., 2011. On the segmentation of 3d lidar point clouds. In: Robotics and Automation (ICRA), 2011 IEEE International Conference on, IEEE, pp. 2798-2805. 

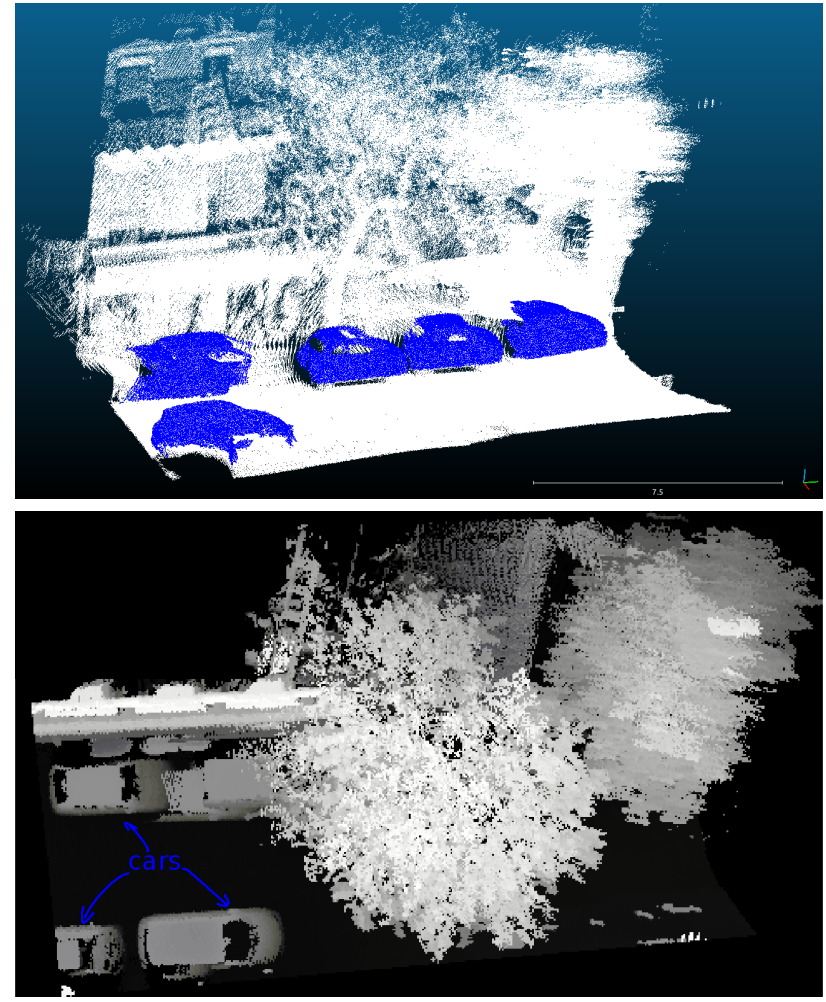

Figure 7: Example of situation where the method of (Serna and Marcotegui, 2014) does not allow to segment cars located under a tree. Up: 3D point cloud with cars well segmented (in blue), down: max elevation image of the same point cloud where cars located under the tree do not appear.

Ferguson, D., Darms, M., Urmson, C. and Kolski, S., 2008. Detection, prediction, and avoidance of dynamic obstacles in urban environments. In: Intelligent Vehicles Symposium, 2008 IEEE, IEEE, pp. 1149-1154.

Golovinskiy, A., Kim, V. G. and Funkhouser, T., 2009. Shapebased recognition of $3 \mathrm{~d}$ point clouds in urban environments. In: Computer Vision, 2009 IEEE 12th International Conference on, IEEE, pp. 2154-2161.

Gorte, B., 2007. Planar feature extraction in terrestrial laser scans using gradient based range image segmentation. In: ISPRS Workshop on Laser Scanning, pp. 173-177.

Goulette, F., Nashashibi, F., Abuhadrous, I., Ammoun, S. and Laurgeau, C., 2006. An integrated on-board laser range sensing system for on-the-way city and road modelling. The International Archives of the Photogrammetry, Remote Sensing and Spatial Information Sciences.

Hoover, A., Jean-Baptiste, G., Jiang, X., Flynn, P. J., Bunke, H., Goldgof, D. B., Bowyer, K., Eggert, D. W., Fitzgibbon, A. and Fisher, R. B., 1996. An experimental comparison of range image segmentation algorithms. Pattern Analysis and Machine Intelligence, IEEE Transactions on 18(7), pp. 673-689.

Kammel, S., Ziegler, J., Pitzer, B., Werling, M., Gindele, T., Jagzent, D., Schröder, J., Thuy, M., Goebl, M., Hundelshausen, F. v. et al., 2008. Team annieway's autonomous system for the 2007 darpa urban challenge. Journal of Field Robotics 25(9), pp. 615639.

Lalonde, J.-F., Unnikrishnan, R., Vandapel, N. and Hebert, M., 2005. Scale selection for classification of point-sampled 3d surfaces. In: 3-D Digital Imaging and Modeling, 2005. 3DIM 2005. Fifth International Conference on, IEEE, pp. 285-292.
Lodha, S. K., Fitzpatrick, D. M. and Helmbold, D. P., 2007. Aerial lidar data classification using adaboost. In: 3-D Digital Imaging and Modeling, 2007. 3DIM'07. Sixth International Conference on, IEEE, pp. 435-442.

Marton, Z.-C., Pangercic, D., Blodow, N., Kleinehellefort, J. and Beetz, M., 2010a. General 3d modelling of novel objects from a single view. In: Intelligent Robots and Systems (IROS), 2010 IEEE/RSJ International Conference on, IEEE, pp. 3700-3705.

Marton, Z.-C., Pangercic, D., Rusu, R. B., Holzbach, A. and Beetz, M., 2010b. Hierarchical object geometric categorization and appearance classification for mobile manipulation. In: Humanoid Robots (Humanoids), 2010 10th IEEE-RAS International Conference on, IEEE, pp. 365-370.

Mountrakis, G., Im, J. and Ogole, C., 2011. Support vector machines in remote sensing: A review. ISPRS Journal of Photogrammetry and Remote Sensing 66(3), pp. 247-259.

Munoz, D., Vandapel, N. and Hebert, M., 2009. Onboard contextual classification of 3-d point clouds with learned high-order markov random fields. In: Robotics and Automation, 2009. ICRA'09. IEEE International Conference on, IEEE.

Osada, R., Funkhouser, T., Chazelle, B. and Dobkin, D., 2001. Matching $3 \mathrm{~d}$ models with shape distributions. In: Shape Modeling and Applications, SMI 2001 International Conference on., IEEE, pp. 154-166.

Pedregosa, F., Varoquaux, G., Gramfort, A., Michel, V., Thirion, B., Grisel, O., Blondel, M., Prettenhofer, P., Weiss, R., Dubourg, V., Vanderplas, J., Passos, A., Cournapeau, D., Brucher, M., Perrot, M. and Duchesnay, E., 2011. Scikit-learn: Machine learning in Python. Journal of Machine Learning Research 12, pp. 28252830.

Rusu, R. B. and Cousins, S., 2011. 3D is here: Point Cloud Library (PCL). In: IEEE International Conference on Robotics and Automation (ICRA), Shanghai, China.

Rusu, R. B., Bradski, G., Thibaux, R. and Hsu, J., 2010. Fast 3d recognition and pose using the viewpoint feature histogram. In: Intelligent Robots and Systems (IROS), 2010 IEEE/RSJ International Conference on, IEEE, pp. 2155-2162.

Rusu, R. B., Holzbach, A., Beetz, M. and Bradski, G., 2009. Detecting and segmenting objects for mobile manipulation. In: Computer Vision Workshops (ICCV Workshops), 2009 IEEE 12th International Conference on, IEEE, pp. 47-54.

Serna, A. and Marcotegui, B., 2014. Detection, segmentation and classification of $3 \mathrm{~d}$ urban objects using mathematical morphology and supervised learning. ISPRS Journal of Photogrammetry and Remote Sensing 93, pp. 243-255.

Velizhev, A., Shapovalov, R. and Schindler, K., 2012. Implicit shape models for object detection in $3 \mathrm{~d}$ point clouds. In: International Society of Photogrammetry and Remote Sensing Congress, Vol. 2.

Wohlkinger, W. and Vincze, M., 2011. Ensemble of shape functions for $3 \mathrm{~d}$ object classification. In: Robotics and Biomimetics (ROBIO), 2011 IEEE International Conference on, IEEE, pp. 2987-2992.

Zhu, X., Zhao, H., Liu, Y., Zhao, Y. and Zha, H., 2010. Segmentation and classification of range image from an intelligent vehicle in urban environment. In: Intelligent Robots and Systems (IROS), 2010 IEEE/RSJ International Conference on, IEEE, pp. 1457-1462. 